\title{
Prevalence and associated factors for pterygium in a Chinese rural population with type 2 diabetes in a cross-sectional study: Jiangsu Diabetic Eye Disease Study (JDEDS)
}

Junfang Zhang ( $\sim$ junfangzh@126.com )

Affiliated Hospital of Nantong University https://orcid.org/0000-0002-4056-6928

\section{Bai Qin}

Affiliated Hospital of Nantong University

Bihong Liu

Funing Shizhuang Eye Hospital

Dajun Sun

Funing Shizhuang Eye Hospital

\section{Congkai Liang}

Funing Country Center for Disease Prevention and Control, Yancheng

\section{Shiyi Wang}

Affiliated Hospital of Nantong University

\section{Mei Yang}

Affiliated Hospital of Nantong University

\section{Rongrong Zhu}

Affiliated Hospital of Nantong University https://orcid.org/0000-0002-3519-7566

\section{Research Article}

Keywords: pterygium, prevalence, type 2 diabetes(D2M), Body mass index (BMI), glycosylated hemoglobin (HbA1c), cigarette smoking

Posted Date: March 8th, 2022

DOI: https://doi.org/10.21203/rs.3.rs-1357976/v1

License: (c) (i) This work is licensed under a Creative Commons Attribution 4.0 International License. Read Full License 


\section{Abstract}

Purpose: To investigate the pterygium prevalence and evaluate risk factors of pterygium in rural community-based type 2 diabetic (D2M) patients aged 50 years and above in Funing Country, Jiangsu Province, China.

Methods A cross-sectional ophthalmic survey was conducted in type 2 diabetes mellitus (D2M) patients aged $\geq 50$ years in Funing county, Jiangsu Province, China, which was named Jiangsu Diabetic Eye Disease Study (JDEDS). All participants underwent a comprehensive questionnaire and ocular examination. Pterygium was diagnosed by slit lamp examination. The risk factors associated with pterygium were evaluated with logistic regression models.

Results The prevalence of pterygium was $22.37 \%(n=427)$ and $95 \%$ confidence interval $(\mathrm{Cl})$ : $(20.50-24.24 \%)$ in D2M patients aged 50 years and above in JDEDS. The prevalence of pterygium was $18.32 \%$ (95\% Cl: $15.33 \sim 21.32 \%$ ) in men and $24.43 \%$ (95\% Cl: $22.06 ~ 26.80 \%)$ in women. Women had a higher prevalence than men ( $p=0.001)$. Multivariate analysis showed, for male participants with D2M, pterygium was independently associated with increasing age [ 70-79yrs: OR and 95\% Cl: 2.49(1.20 5.18), $p=0.014 ; \geq 80 y r s: 4.84(2.04 \sim 11.47), p<0.001]$, while cigarette smoking was the protective factors, especially in current smoker [OR and 95\% Cl: 0.79(0.67 0.92); $p=0.003$ ]. For female participants with $\mathrm{D} 2 \mathrm{M}$, age [ $60-69 \mathrm{yrs}$ OR and $95 \% \mathrm{Cl}$ : 1.68(1.07 2.62), $p=0.023$; 70-79yrs: 2.62(1.69 4.06), $p<0.001$; $\geq 80 \mathrm{yrs}: 3.24(1.70 \sim 5.90), p<0.001]$, hypertension [OR and 95\%Cl: 1.40(1.05 1.87), $p=0.024$ ], BMI 24-27.9 [OR and 95\% Cl: 1.20(1.00 1.44), $p=0.047$ ], higher HbA1c [ (5.6-7.9) \% OR and 95\%Cl: 1.42(1.10 1.82), $p=0.006$; (8.0-9.9) \%: 1.32(1.10 1.58), $p=0.003]$ were risk factors.

Conclusions D2M patients aged over 50 years has a high prevalence of pterygium in JDEDS. The pterygium prevalence is higher in female D2M participants. Diabetes and related factors may be risk factors of pterygium in female D2M patients. Further studies are needed to explore the gender difference in the pathogenesis of pterygium in D2M subjects.

\section{Highlights}

1. The present firstly report the pterygium prevalence was $22.37 \%$ in rural D2M population aged 50 years and above.

2. Women had a higher prevalence than men with D2M in JDEDS.

3. Diabetic factors such as higher BMI and HbA1c were risk factors of pterygium only for female participants with D2M.

4. Smoking was a protective factor of pterygium, especially for male participants with D2M currently smoking.

\section{Background}

Diabetes mellitus (DM) is a serious public health problem in many countries. Over the last three decades, the number of diabetic patients has more than doubled due to lifestyle changes and their consequences, such as obesity and a sedentary lifestyle. The prevalence of diabetes was $11.2 \%$ among adults living in China according to the WHO criteria[1]. Type 2 diabetes (D2M) is the most common type of diabetes, accounting for around $90 \%$ of all diabetes cases. DM can cause a variety of ocular complications, including diabetic retinopathy, cataracts, glaucoma, keratopathy, dry eye disease (DED) and so on. Pterygium is not a common complication of DM, but it is a cause of unilateral low vision and blindness in D2M patients[2].

Pterygium is a common chronic external ocular disease affecting humans only. It is characterized by a wing-shaped fibrovascular proliferative tissue, growth from the nasal or temporal limbus over the clear cornea. The prevalence of pterygia has been assessed in numerous previous investigations, which were carried out in various countries and different provinces in China[2-14]. The prevalence of pterygium was $12 \%$ in the whole world[15]. The lowest prevalence of pterygium was reported in a clinic-based study in Saudi Arabia (0.07\%) [16]and the highest prevalence was in the Brazilian Amazon Region (58.8\%) [14]. The marked differences in the pterygium prevalence in the various investigations may be due to differences in the study populations, including differences in age and gender, the lifestyle and exposure to ultraviolet light, and geographic and 
socioeconomic differences between the various regions. Most of these studies consistently revealed that the main risk factors were older age, and ultraviolet light (UV) exposure and its associated factors such as low education, outdoor work, without glasses and hat, and habitation in low latitude and rural area. However, conflicting data regarding gender and smoking on pterygium have been reported.

The associations of the pterygium prevalence with other systemic diseases like DM were explored in several studies. Some studies reported DM was not a risk factor of pterygium in general population[17-19]. However, several recent studies showed DM and related factors such as higher blood glucose and high density lipoprotein (HDL) level were risk factors of pterygium[10, 20, 21].Nam et al reported that higher body mass index (BMI) and waist circumference(WC) were positively associated with the risk of pterygium in obese Korean women[22]. However, little is known about the prevalence of pterygium in diabetic population. Oure study explored whether D2M and associated factors were risk factors of pterygium and affected the prevalence of pterygium.

The Jiangsu Diabetic Eye Disease Study (JDEDS) was a cross-sectional ophthalmic survey conducted in a Chinese rural population with D2M, which was designed to estimate the general prevalence of eye diseases related to diabetes and its associated risk factors among a native country with D2M residing in rural areas of Jiangsu province, eastern of China. The present study was a part of JDEDS, in order to evaluate the prevalence of pterygium in a Chinese rural population with D2M aged 50 years and above, and explore whether diabetes related factors were risk to the pterygium.

\section{Materials And Methods Study setting}

The JDEDS was a cross-sectional study using cluster random sampling, carried out between March 2019 and May 2019 in Funing Country, Jiangsu Province, China. Jiangsu is an eastern coastal province located in in the Yangtze River Delta. Funing is an agricultural county in Northern of Jiangsu, at the eastern longitude $116^{\circ}$ to $121^{\circ}$ and northern latitude $30^{\circ}$ to $35^{\circ}$. A population-based epidemiological study of eye diseases was conducted in Funing County in 2011. The prevalence of pterygium was $30.97 \%$ in people aged 50 years and above in Funing County, which was much higher than the general prevalence of pterygium in China at $13.4 \% .[23,24]$

\section{Sample size}

The sample size was calculated using the following formula: $N=Z^{2}(p \llbracket 1-p) / B^{2}$. Sample size was based on estimating an anticipated $18.2 \%$ prevalence for diabetic retinopathy within an error bound (precision) of $20 \%$ with $95 \%$ confidence interval. The corresponding error value $B^{2}=(0.182 \times 0.15)^{2}=0.0007$, the sampling coefficient of 2 was used to correct the sampling error. The calculated sample size was 1887 . The prevalence rate of diabetes in Funing county was $2.3 \%$ (the number of diabetic patients provided by the Centers for Disease Control and prevention in Funing county to the total population of Funing county). According to the China Nine-Province Survey[25], the basic sample unit (BSU) was constructed using geographically defined clusters based on village register data. Each BSU would have a population of approximately 1000 individuals (all ages). It was estimated that 82 BSU was needed, of which two BSUs were used tpre tested and a total of 84 BSU were sampled.

\section{Inclusive and exclusive criteria}

The following were the inclusive criteria: (1) all participants were diagnosed with D2M by physician (either fasting plasma glucose $\geq 126 \mathrm{mg} / \mathrm{dL}$, or random glucose $\geq 200 \mathrm{mg} / \mathrm{dL}$ or $\mathrm{HbA} 1 \mathrm{C} \geq 6.5 \%$ ); (2) D2M patients registered in Funing Country Center for Chronic Disease Control and Prevention; (3) age $\geq 50$ years; (4) residents who had been living in Funing country for more than six months; (5) underwent ophthalmic examinations and (6) finished all information in the questionnaire.

The following were the exclusive criteria. (1) D2M patients who have left the survey site for more than half a year; (2) D2M patients not registered in Funing Country Center for Chronic Disease Control and Prevention. 


\section{Data collection and examinations}

A comprehensive interview with unified questionnaires was held by trained interviewers to obtain demographic (age, gender, birth date, race, education level, income), UV-related information (sun exposure, exercise), lifestyle (smoking, alcohol consumption and mobilephone use) and medical history (hypertension, hyperlipidemia, diabetic nephropathy, diabetic foot, et al). Agriculture work was considered as outdoor occupation, while other occupations were considered as indoor. Smoking/drinking status was recorded as never, ever, and current.

\section{General systemic examination and Ophthalmic examination}

A systemic examination included the anthropomorphic parameters of body height, body weight, blood pressure, fasting blood sample collection and ophthalmic examination. Systolic blood pressures (SBPs) and diastolic blood pressures (DBPs) were measured with a digital automatic blood pressure monitor after the participants were seated for over 10 min. Fasting venous blood samples were collected for biochemistry tests, including fasting blood glucose (FBG) and glycolate hemoglobin (HbA1c). Individuals with a hypertension history or whose SBP $\geq 140 \mathrm{~mm} \mathrm{Hg}$ or DBP $\geq 90 \mathrm{~mm} \mathrm{Hg}$ were defined as hypertension.

The ophthalmic examination included visual acuity, slit-lamp examination, and fundus examination. Naked vision and daily life visual acuity were measured with an ETDRS (Early Treatment Diabetic Retinopathy Study) chart (Wehen, Guangzhou, China) at $4 \mathrm{~m}$. The anterior segment of the eye was examined with a slit-lamp microscope (TopconSL-2F, Topcon, Tokyo, Japan). Lens opacities were graded with Lens Opacities Classification System II in all participants. Lens Opacities Classification System score $\geq 2$ was considered as significant lens opacity. Fundus examination was conducted and taken photos with mydriasis free fundus camera (RetiCam 3100, Chongqing, China).

\section{Diagnosis of Pterygium}

The examination of the anterior segment was performed by two experienced ophthalmologists using a slit lamp microscope. Pterygium was defined as a raised fleshy triangular fibrovascular tissue growth of the conjunctiva encroaching onto the clear cornea. Patients with pterygium in the present study were defined as (1) having pterygium at the time of survey or (2) having pterygium surgery performed and (3) no pterygium at the time of survey. The pterygium was not graded in this study.

\section{Statistics Analysis}

The collected data were double entered into the computer by Epi 3.1 software (Centers for Disease Control and Prevention, Atlanta, GA, USA), then the consistency was tested. All statistical analysis was carried out by Stata 15.0 (StataCorp, College Station, TX, USA). The prevalence rates, odds ratios (OR) and 95\% Confidence intervals (CI) of pterygium were calculated. For independent samples, Student's t-tests were used to compare means, and the Chi-square test was used to compare proportions in different groups. Logistic regression was used to analyzed associations of pterygium and general risk factors, such as age, gender, education, status of smoking and drinking, and hypertension and hyperlipidemia history and diabetic parameters. Multivariate logistic regression models were used to determine independent associated risk factors. $P$ values $\leq 0.05$ was considered statistically significant by two sides test.

\section{Results}

\section{Pterygium prevalence and demographic characteristics}

Among 2067 diabetic participants included in this study, 1965 underwent ophthalmic examinations and 1909 (644 men and 1265 women) had complete information in the questionnaire finally (response rate $=92.4 \%$ ). The average age was $(68.39 \pm$ 8.61) years, ranged from 50 to 95 years old. Among them, $99.32 \%$ (1 896) subjects were farmers and $98.17 \%$ (1 874) were not used to wearing glasses and hats outdoor. Table 1 showed the characteristics of the study population. 
Table 1

The prevalence and associations of pterygium (univariate and multivariable analysis) with systemic parameters in the JDEDS.

\begin{tabular}{|c|c|c|c|c|c|c|c|}
\hline & Case/n & $\begin{array}{l}\text { Prevalence rate } \\
(95 \% \mathrm{Cl})(\%)\end{array}$ & $\begin{array}{l}P \\
\text { value }\end{array}$ & $\mathrm{OR}^{\mathrm{I}}(95 \% \mathrm{Cl})$ & $\begin{array}{l}P \\
\text { value }\end{array}$ & $\begin{array}{l}\text { adjusted } \\
\mathrm{OR}^{\dagger}(95 \% \mathrm{Cl})\end{array}$ & $\begin{array}{l}P \\
\text { value }\end{array}$ \\
\hline Total & $427 / 1909$ & $\begin{array}{l}22.37(20.50 \sim \\
24.24)\end{array}$ & & & & & \\
\hline Age & & & 0.000 & & & & \\
\hline $50-59$ & $41 / 328$ & $12.5(8.91 \sim 16.09)$ & & 1.00 & & 1.00 & \\
\hline $60-69$ & $139 / 705$ & $\begin{array}{l}19.72(16.78 \sim ~ \\
22.66)\end{array}$ & & $\begin{array}{l}1.72(1.18 \sim \\
2.50)\end{array}$ & 0.005 & $1.74(1.19 \sim 2.53)$ & 0.004 \\
\hline $70-79$ & $187 / 692$ & $\begin{array}{l}27.02(23.71 \sim \\
30.34)\end{array}$ & & $\begin{array}{l}2.59(1.79 \sim \\
3.74)\end{array}$ & 0.000 & $2.61(1.81 \sim 3.78)$ & 0.000 \\
\hline$\geq 80$ & $60 / 184$ & $\begin{array}{l}32.61(25.81 \sim \\
39.40)\end{array}$ & & $\begin{array}{l}3.39(2.16 \sim \\
5.31)\end{array}$ & 0.000 & $3.42(2.18 \sim 5.36)$ & 0.000 \\
\hline Gender & & & 0.002 & & & & \\
\hline Male & $118 / 664$ & $\begin{array}{l}18.32(15.33 \sim \\
21.32)\end{array}$ & & 1.00 & & 1.00 & \\
\hline Female & $309 / 1265$ & $\begin{array}{l}24.43(22.06 \sim \\
26.80)\end{array}$ & & $\begin{array}{l}1.44(1.14 \sim \\
1.83)\end{array}$ & 0.003 & $1.46(1.15 \sim 1.86)$ & 0.002 \\
\hline Education & & & 0.000 & & & & \\
\hline No education & $246 / 922$ & $\begin{array}{l}26.68(23.82 \sim \\
29.54)\end{array}$ & & 1.00 & & 1.00 & \\
\hline Elementary & $116 / 564$ & $\begin{array}{l}2056(17.22 \sim \\
23.91)\end{array}$ & & $\begin{array}{l}0.71(0.55 \sim \\
0.91)\end{array}$ & 0.008 & $0.84(0.64 \sim 1.11)$ & 0.229 \\
\hline Middle & $50 / 316$ & $\begin{array}{l}15.82(11.79 \sim \\
19.86)\end{array}$ & & $\begin{array}{l}0.51(0.37 \sim \\
0.72)\end{array}$ & 0.000 & $0.67(0.46 \sim 0.97)$ & 0.035 \\
\hline $\begin{array}{l}\text { Senior and } \\
\text { Higher }\end{array}$ & $15 / 107$ & $14.02(7.41 \sim 20.63)$ & & $\begin{array}{l}0.44(0.25 \sim ~ \\
0.79)\end{array}$ & 0.005 & $0.66(0.36 \sim 1.20)$ & 0.174 \\
\hline Smoking & & & 0.000 & & & & \\
\hline Never & $317 / 1239$ & $\begin{array}{l}25.59(23.15 \sim \\
28.02)\end{array}$ & & 1.00 & & 1.00 & \\
\hline Ever & $44 / 233$ & $\begin{array}{l}18.89(13.84 \sim \\
23.92)\end{array}$ & & $\begin{array}{l}0.82(0.69 \sim \\
0.98)\end{array}$ & 0.030 & $0.82(0.68 \sim 0.98)$ & 0.032 \\
\hline Current & $66 / 437$ & $\begin{array}{l}15.10(11.74 \sim \\
118.47)\end{array}$ & & $\begin{array}{l}0.80(0.72 \sim \\
0.88)\end{array}$ & 0.000 & $0.82(0.74 \sim 0.91)$ & 0.000 \\
\hline Alcohol & & & 0.059 & & & & \\
\hline Never & $326 / 1371$ & $\begin{array}{l}23.78(21.52 \sim \\
26.03)\end{array}$ & & 1.00 & & 1.00 & \\
\hline Ever & $39 / 200$ & $\begin{array}{l}18.77(15.47 \sim \\
22.08)\end{array}$ & & $\begin{array}{l}0.88(0.73 \sim \\
1.06)\end{array}$ & 0.182 & $0.94(0.77 \sim 1.14)$ & 0.513 \\
\hline Current & $62 / 338$ & $\begin{array}{l}18.34(14.21 \sim \\
22.48)\end{array}$ & & $\begin{array}{l}0.90(0.81 \sim \\
0.99)\end{array}$ & 0.033 & $0.97(0.87 \sim 1.09)$ & 0.648 \\
\hline Exercise & & & 0.094 & & & & \\
\hline
\end{tabular}

Notes: $\otimes$ is univariate analysis and $\dagger$ is multivariable analysis. 


\begin{tabular}{|c|c|c|c|c|c|c|c|}
\hline & Case/n & $\begin{array}{l}\text { Prevalence rate } \\
(95 \% \mathrm{Cl})(\%)\end{array}$ & $\begin{array}{l}P \\
\text { value }\end{array}$ & $\mathrm{OR}^{\mathrm{Q}}(95 \% \mathrm{Cl})$ & $\begin{array}{l}P \\
\text { value }\end{array}$ & $\begin{array}{l}\text { adjusted } \\
\mathrm{OR}^{\dagger}(95 \% \mathrm{Cl})\end{array}$ & $\begin{array}{l}P \\
\text { value }\end{array}$ \\
\hline Not regular & $174 / 712$ & $\begin{array}{l}24.44(21.28 \sim \\
27.60)\end{array}$ & & 1.00 & & 1.00 & \\
\hline Regular & $253 / 1197$ & $\begin{array}{l}21.14(18.82 \sim \\
23.45)\end{array}$ & & $\begin{array}{l}0.83(0.66 \sim \\
1.03)\end{array}$ & 0.094 & $0.97(0.77 \sim 1.22)$ & 0.819 \\
\hline $\begin{array}{l}\text { Mobile phone } \\
\text { use }\end{array}$ & & & 0.000 & & & & \\
\hline Seldom & $60 / 417$ & $\begin{array}{l}14.39(11.01 \sim \\
17.76)\end{array}$ & & 1.00 & & 1.00 & \\
\hline Often & $367 / 1492$ & $\begin{array}{l}24.60(22.41 \sim \\
26.79)\end{array}$ & & $\begin{array}{l}0.51(0.38 \sim \\
0.69)\end{array}$ & 0.000 & $0.75(0.54 \sim 1.03)$ & 0.079 \\
\hline Sun exposure & & & 0.017 & & & & \\
\hline$<5 h$ & $73 / 406$ & $\begin{array}{l}17.98(14.24 \sim \\
21.72)\end{array}$ & & 1.00 & & 1.00 & \\
\hline$\geq 5 \mathrm{~h}$ & $354 / 1503$ & $\begin{array}{l}23.55(21.41 \sim \\
25.70)\end{array}$ & & $\begin{array}{l}1.41(1.06 \sim \\
1.86)\end{array}$ & 0.017 & $1.42(1.07 \sim 1.88)$ & 0.016 \\
\hline Hypertension & & & 0.015 & & & & \\
\hline No & $145 / 745$ & $\begin{array}{l}19.46(16.61 \sim \\
22.31)\end{array}$ & & 1.00 & & 1.00 & \\
\hline Yes & $282 / 1164$ & $\begin{array}{l}24.23(21.76 \sim \\
26.69)\end{array}$ & & $\begin{array}{l}1.32(1.06 \sim \\
1.66)\end{array}$ & 0.015 & $1.23(0.98 \sim 1.55)$ & 0.077 \\
\hline Hyperlipidemia & & & 0.657 & & & & \\
\hline No & $287 / 1266$ & $\begin{array}{l}22.67(20.36 \sim \\
24.98)\end{array}$ & & 1.00 & & 1.00 & \\
\hline Yes & $140 / 643$ & $\begin{array}{l}21.77(18.57 \sim \\
24.97)\end{array}$ & & $\begin{array}{l}0.94(0.76 \sim \\
1.19)\end{array}$ & 0.657 & $0.99(0.78 \sim 1.24)$ & 0.906 \\
\hline
\end{tabular}

Pterygium was diagnosed in 427 subjects (22.37\%, 95\% Cl: 20.50\% 24.24\%), 171 (8.96\%) were bilateral and 256 (13.41\%) were unilateral. Among them, 45(2.36\%) participants underwent pterygium removal surgery. The prevalence of pterygium was 18.32 (95\% Cl:15.33 21.32) in men and 24.43 (95\% Cl:22.06 26.80) in women. The prevalence rate increased obviously with ages in both men and women $(p<0.001)$. However, the prevalence of pterygium in women was apparently higher than that in men $(p=0.007)$ and the difference was still significant after the age adjusted $(p=0.002)$. The prevalence rates for pterygium by age group, gender, systemic factors and D2M related factors and complication were shown in detail in Table 1.

\section{General risk factors of pterygium}

The univariate analysis for the prevalence of pterygium showed that older age $(p=0.000)$, female $(p=0.000)$, lower education $(p<0.001)$, sun exposure $(p=0.017)$ and hypertension $(p=0.015)$, mobile phone use $(p<0.001)$ were risk factors of pterygium, while smoking $(p<0.001)$ were protective factors. After adjusted by age and gender, lower education $(p=0.035)$, sun exposure $(p=0.016)$ was still risk factors and smoking was protective factor. The prevalence, ORs and $95 \% \mathrm{Cl}$ of the risk factors for pterygium were shown in Table 1.

Among diabetes related factors and complications, blood glucose levels was significantly associated with pterygium. DN and nuclear opacity grade over 2 showed the association with pterygium, but lose their significance after age and gender adjusted. The other factors and complications did not show the associations with pterygium. The data were shown in Table 2. 
Table 2

Effects of associated factors and complications of D2M on the prevalence of pterygium.

\begin{tabular}{|c|c|c|c|c|c|c|c|c|c|}
\hline & Total & & Male & & & & Female & & \\
\hline & Case/n & $\begin{array}{l}\text { Prevalence } \\
\text { rate }\end{array}$ & $\begin{array}{l}P \\
\text { value }\end{array}$ & Case/n & $\begin{array}{l}\text { Prevalence } \\
\text { rate }\end{array}$ & $\begin{array}{l}P \\
\text { value }\end{array}$ & Case/n & $\begin{array}{l}\text { Prevalence } \\
\text { rate }\end{array}$ & $\begin{array}{l}P \\
\text { value }\end{array}$ \\
\hline & & $(95 \% \mathrm{Cl})(\%)$ & & & $(95 \% \mathrm{Cl})(\%)$ & & & $(95 \% \mathrm{Cl})(\%)$ & \\
\hline BMI & & & 0.120 & & & 0.855 & & & 0.071 \\
\hline$<24$ & $95 / 451$ & $\begin{array}{l}21.06(17.29 \\
\sim 24.83)\end{array}$ & & $29 / 156$ & $\begin{array}{l}\text { 18.59(12.45 } \\
\sim 24.73)\end{array}$ & & $66 / 295$ & $\begin{array}{l}\text { 22.37(17.60 } \\
\sim 21.74)\end{array}$ & \\
\hline $24-27.9$ & $207 / 843$ & $\begin{array}{l}24.56(21.65 \\
\sim 27.46)\end{array}$ & & $57 / 300$ & $\begin{array}{l}19.00(14.54 \\
\sim 23.46)\end{array}$ & & $150 / 543$ & $\begin{array}{l}\text { 27.62(23.85 } \\
\sim 31.39)\end{array}$ & \\
\hline$\geq 28$ & $125 / 615$ & $\begin{array}{l}\text { 20.32(17.14 } \\
\sim 23.51)\end{array}$ & & $32 / 188$ & $\begin{array}{l}\text { 17.02(11.62 } \\
\sim 22.42)\end{array}$ & & $93 / 427$ & $\begin{array}{l}\text { 21.78(17.86 } \\
\sim 25.70)\end{array}$ & \\
\hline $\begin{array}{l}\text { Blood } \\
\text { glucose }\end{array}$ & & & 0.038 & & & 0.099 & & & 0.196 \\
\hline$<6.1$ & $17 / 92$ & $\begin{array}{l}18.48(10.50 \\
\sim 26.46)\end{array}$ & & $6 / 35$ & $\begin{array}{l}17.14(4.45 \\
\sim 29.83)\end{array}$ & & $11 / 57$ & $\begin{array}{l}19.30(8.95 \\
\sim 29.64)\end{array}$ & \\
\hline $6.1-7.9$ & $197 / 780$ & $\begin{array}{l}25.26(22.20 \\
\sim 28.31)\end{array}$ & & $60 / 271$ & $\begin{array}{l}\text { 22.14(17.18 } \\
\sim 27.10)\end{array}$ & & $137 / 509$ & $\begin{array}{l}26.92(23.06 \\
\sim 30.78)\end{array}$ & \\
\hline$\geq 8.0$ & $213 / 1,087$ & $\begin{array}{l}20.54(18.08 \\
\sim 23.00)\end{array}$ & & $52 / 338$ & $\begin{array}{l}\text { 15.38(11.53 } \\
\sim 19.24)\end{array}$ & & $161 / 699$ & $\begin{array}{l}\text { 23.03(19.91 } \\
\sim 26.16)\end{array}$ & \\
\hline $\begin{array}{l}\mathrm{HbA1c} \\
(\%)\end{array}$ & & & 0.258 & & & 0.978 & & & 0.136 \\
\hline$<5.6$ & $43 / 229$ & $\begin{array}{l}\text { 18.78(13.70 } \\
\sim 23.85)\end{array}$ & & $16 / 82$ & $\begin{array}{l}\text { 19.51(10.87 } \\
\sim 28.16)\end{array}$ & & $27 / 147$ & $\begin{array}{l}18.37(12.08 \\
\sim 24.65)\end{array}$ & \\
\hline $5.6-7.9$ & $206 / 876$ & $\begin{array}{l}23.52(20.70 \\
\sim .26 .33)\end{array}$ & & $51 / 274$ & $\begin{array}{l}18.61(13.99 \\
\sim 23.24)\end{array}$ & & $155 / 602$ & $\begin{array}{l}25.74(22.25 \\
\sim 29.25)\end{array}$ & \\
\hline $8.0-9.9$ & $110 / 463$ & $\begin{array}{l}\text { 23.76(19.87 } \\
\sim 27.64)\end{array}$ & & $30 / 166$ & $\begin{array}{l}\text { 18.07(12.19 } \\
\sim 23.95)\end{array}$ & & $80 / 297$ & $\begin{array}{l}\text { 26.94(21.88 } \\
\sim 31.99)\end{array}$ & \\
\hline$\geq 10.0$ & $68 / 341$ & $\begin{array}{l}\text { 19.94(15.69 } \\
\sim 24.19)\end{array}$ & & $21 / 122$ & $\begin{array}{l}17.21(10.47 \\
\sim 23.95)\end{array}$ & & $47 / 219$ & $\begin{array}{l}21.46(16.01 \\
\sim 26.92)\end{array}$ & \\
\hline $\begin{array}{l}\text { Family } \\
\text { history }\end{array}$ & & & 0.057 & & & 0.240 & & & 0.153 \\
\hline No & $364 / 1,568$ & $\begin{array}{l}23.21(21.12 \\
\sim 25.31)\end{array}$ & & $100 / 521$ & $\begin{array}{l}19.19(15.80 \\
\sim 22.59)\end{array}$ & & $264 / 1,047$ & $\begin{array}{l}25.21(22.58 \\
\sim 27.85)\end{array}$ & \\
\hline Yes & $63 / 341$ & $\begin{array}{l}\text { 18.48(14.35 } \\
\sim 22.60)\end{array}$ & & $18 / 123$ & $\begin{array}{l}14.63(8.35 \\
\sim 20.92)\end{array}$ & & $45 / 218$ & $\begin{array}{l}20.64(15.25 \\
\sim 26.03)\end{array}$ & \\
\hline $\begin{array}{l}\text { Diabetic } \\
\text { course }\end{array}$ & & & 0.284 & & & 0.816 & & & 0.274 \\
\hline$\leq 10$ & $339 / 1490$ & $\begin{array}{l}\text { 23.61(20.84 } \\
\sim 26.39)\end{array}$ & & $98 / 522$ & $\begin{array}{l}\text { 18.77(15.41 } \\
\sim 22.13)\end{array}$ & & $241 / 968$ & $\begin{array}{l}24.90(22.17 \\
\sim 27.62)\end{array}$ & \\
\hline $11-20$ & $81 / 367$ & $\begin{array}{l}21.43(18.11 \\
\sim 24.75)\end{array}$ & & $17 / 102$ & $\begin{array}{l}16.67(9.38 \\
\sim 23.95)\end{array}$ & & $64 / 265$ & $\begin{array}{l}24.15(18.98 \\
\sim 29.32)\end{array}$ & \\
\hline
\end{tabular}

Notes: BMI: Body mass index = weight $(\mathrm{kg}) /$ height $\left(\mathrm{m}^{2}\right)$; HbA1c: glycosylated hemoglobin; DR: Diabetic retinopathy; DME: diabetic macular edema; DN: Diabetic nephropathy; DF: Diabetic foot; Nuclear grade classified by LOCS $₫$ 


\begin{tabular}{|c|c|c|c|c|c|c|c|c|c|}
\hline & Total & & Male & & & & Female & & \\
\hline$>20$ & $7 / 52$ & $\begin{array}{l}13.46(4.09 \\
\sim 22.83)\end{array}$ & & $3 / 20$ & $\begin{array}{l}15.00(-1.09 \\
\sim 31.09)\end{array}$ & & $4 / 32$ & $\begin{array}{l}12.50(0.84 \\
\sim 24.15)\end{array}$ & \\
\hline Cure & & & 0.152 & & & 0.388 & & & 0.292 \\
\hline $\begin{array}{l}\text { Diet } \\
\text { control }\end{array}$ & $144 / 571$ & $\begin{array}{l}\text { 25.22(21.65 } \\
\sim 28.79)\end{array}$ & & $42 / 187$ & $\begin{array}{l}22.46(16.45 \\
\sim 28.47)\end{array}$ & & $102 / 384$ & $\begin{array}{l}26.56(22.14 \\
\sim 30.99)\end{array}$ & \\
\hline $\begin{array}{l}\text { Oral } \\
\text { drug }\end{array}$ & $231 / 1,058$ & $\begin{array}{l}\text { 21.83(19.34 } \\
\sim 24.33)\end{array}$ & & $80 / 363$ & $\begin{array}{l}\text { 16.53(12.70 } \\
\sim 20.36)\end{array}$ & & $171 / 695$ & $\begin{array}{l}24.60(21.40 \\
\sim 27.81)\end{array}$ & \\
\hline Insulin & $29 / 150$ & $\begin{array}{l}\text { 19.33(12.99 } \\
\sim 25.68)\end{array}$ & & $9 / 53$ & $\begin{array}{l}16.98(6.76 \\
\sim 27.21)\end{array}$ & & $20 / 97$ & $\begin{array}{l}20.62(12.52 \\
\sim 28.72)\end{array}$ & \\
\hline $\begin{array}{l}\text { Oral } \\
\text { drug + } \\
\text { Insulin }\end{array}$ & $23 / 130$ & $\begin{array}{l}17.69(11.10 \\
\sim 24.28)\end{array}$ & & $7 / 41$ & $\begin{array}{l}17.07(5.39 \\
\sim 28.76)\end{array}$ & & $16 / 89$ & $\begin{array}{l}17.98(9.95 \\
\sim 26.01)\end{array}$ & \\
\hline DN & & & 0.019 & & & 0.211 & & & 0.075 \\
\hline No & $403 / 1,749$ & $\begin{array}{l}23.04(21.07 \\
\sim 25.02\end{array}$ & & $109 / 574$ & $\begin{array}{l}\text { 18.99(15.77 } \\
\sim 22.21)\end{array}$ & & $284 / 1175$ & $\begin{array}{l}\text { 25.02(22.54 } \\
\sim 27.50)\end{array}$ & \\
\hline Yes & $24 / 160$ & $\begin{array}{l}15.00(9.45 \\
\sim 20.55)\end{array}$ & & $9 / 70$ & $\begin{array}{l}12.86(4.94 \\
\sim 20.77)\end{array}$ & & $15 / 90$ & $\begin{array}{l}16.67(8.91 \\
\sim 24.41)\end{array}$ & \\
\hline DF & & & 0.585 & & & 0.627 & & & 0.684 \\
\hline No & $413 / 1,838$ & $\begin{array}{l}22.47(20.56 \\
\sim 24.38)\end{array}$ & & $115 / 623$ & $\begin{array}{l}18.46(15.40 \\
\sim 21.51)\end{array}$ & & $298 / 1,215$ & $\begin{array}{l}\text { 24.53(22.10 } \\
\sim 26.95)\end{array}$ & \\
\hline Yes & $14 / 71$ & $\begin{array}{l}\text { 19.72(10.39 } \\
\sim 29.04)\end{array}$ & & $3 / 21$ & $\begin{array}{l}14.29(-1.07 \\
\sim 29.65)\end{array}$ & & $11 / 50$ & $\begin{array}{l}22.00(10.39 \\
\sim 3361)\end{array}$ & \\
\hline DR & & & 0.407 & & & 0.429 & & & 0.790 \\
\hline No & $268 / 1,161$ & $\begin{array}{l}\text { 23.08(20.66 } \\
\sim 25.51)\end{array}$ & & $88 / 498$ & $\begin{array}{l}\text { 17.67(14.31 } \\
\sim 21.03)\end{array}$ & & $218 / 900$ & $\begin{array}{l}\text { 24.22(21.42 } \\
\sim 27.03)\end{array}$ & \\
\hline Yes & $141 / 659$ & $\begin{array}{l}21.40(18.26 \\
\sim 24.53)\end{array}$ & & $30 / 146$ & $\begin{array}{l}20.54(13.96 \\
\sim 27.14)\end{array}$ & & $91 / 365$ & $\begin{array}{l}24.93(20.48 \\
\sim 29.38)\end{array}$ & \\
\hline DME & & & 0.332 & & & 0.206 & & & 0.314 \\
\hline No & $420 / 1,866$ & $\begin{array}{l}22.51(20.61 \\
\sim 24.40)\end{array}$ & & $117 / 628$ & $\begin{array}{l}18.63(15.58 \\
\sim 21.68)\end{array}$ & & $300 / 1,216$ & $\begin{array}{l}24.67(22.24 \\
\sim 27.10)\end{array}$ & \\
\hline Yes & $7 / 43$ & $\begin{array}{l}16.28(5.11 \\
\sim 27.45)\end{array}$ & & $1 / 16$ & $\begin{array}{l}6.25(-6.02 \sim \\
18.52)\end{array}$ & & $9 / 49$ & $\begin{array}{l}\text { 18.37(7.40 } \\
\sim 29.33)\end{array}$ & \\
\hline $\begin{array}{l}\text { Nuclear } \\
\text { grade }\end{array}$ & & & 0.000 & & & 0.020 & & & 0.001 \\
\hline$\leq 2$ & $224 / 1,177$ & $\begin{array}{l}\text { 19.03(16.79 } \\
\sim 21.28)\end{array}$ & & $75 / 465$ & $\begin{array}{l}\text { 16.13(12.78 } \\
\sim 19.48)\end{array}$ & & $149 / 712$ & $\begin{array}{l}\text { 20.93(17.93 } \\
\sim 23.92)\end{array}$ & \\
\hline$>2$ & $203 / 732$ & $\begin{array}{l}22.73(24.48 \\
\sim 30.98)\end{array}$ & & $43 / 179$ & $\begin{array}{l}24.02(17.73 \\
\sim 30.31)\end{array}$ & & $160 / 553$ & $\begin{array}{l}28.93(25.15 \\
\sim 32.72)\end{array}$ & \\
\hline
\end{tabular}

\section{Multivariate analysis}

The multivariate logistic regression model was based on a univariate analysis, and the following factors included in the final calculation: age, gender, education level, smoking and drinking status, exercise, and sunlight exposure, mobile phone use, 
hypertension, hyperlipemia, BMI, blood glucose, $\mathrm{HbA1c}$, family history, cure methods, DN, DR, DME, DF, nuclear grade. The results showed that being 60-69 years old $(p=0.20), 70-80$ years old $(p<0.001), \geq 80$ years old $(p<0.001)$; often sunlight exposure $(p=0.029)$, hypertension $(p=0.031)$; BMI 24-27.9 $(p=0.054)$ and nuclear grade over $2(p=0.019)$ were risk factors for pterygium. Current smoking $(p<0.001)$ and $D N(p=0.029)$ were protective factors. The results of the logistic regression analysis were presented in Fig. 1.

We also performed a subgroup multivariate logistic regression analysis on male and female participants. For male diabetic participants, only age $\geq 70$ years old was a risk factor (70-80: $p=0.014 ; \geq 80$ : $p<0.001)$, while current smoking was a protective factor $(p=0.003)$. For female diabetic participants, age $\geq 60$ years old was a risk factor $(60-70: p=0.023 ; 70-80$ : $p$ $<0.001 ; \geq 80: p<0.001)$. BMI, HbA1c and hypertension were risk factors for only women with D2M (BMI 24-27.9: $p=0.047$; HbA1 5.6-7.9: $p=0.006$; HbA1 8.0-9.9: $p=0.003)$; hypertension $(p=0.024)$. The results were shown in Fig. 2.

\section{Discussion}

To the best of our knowledge, this was the first study to investigate the prevalence and risk factors of pterygium in a diabetic population. In the present study, the pterygium prevalence was $22.37 \%$ in D2M population aged 50 years and above, which was lower than that in a general population (32.79\%) in Funing and in Dali $(39 \%)$, China[17, 24]. However, the pterygium prevalence was still higher than that reported by Chinese other province studies in Shandong (10.53\%)[19], Hebei (6.5\%)[20, 26], Xinjian (11.95\%)[18], Inner Mongolia (6.4\%)[27] and Gansu(9.3\%)[28]. The high prevalence of pterygium in Funing may be attributed to the following reasons. Firstly, Funing County is located at $33^{\circ} \mathrm{N}$ in "pterygium belt", which is between the latitudes of $37^{\circ} \mathrm{N}$ and $37^{\circ} \mathrm{S}$ has a high prevalence of pterygium[29]. It is a traditional, agriculturally based society and crops are planted and harvested twice a year. Accordingly, the participants exposure to the sunlight for more hours in a year. Secondly, all participants enrolled in our study were over 50 years old and the mean age reached $68.39 \pm 8.61$, higher than others studies. It was reported that prevalence of pterygium was highest among 60-79-year-old individuals[30]. Thirdly, the participants were mainly farmers (99.32\%) with low education (illiteracy) and low income ( $97.07 \% \leq 1500$ yuan) (data not shown). Finally, almost all participants were not accustomed to using protective tools (sunglasses, hats, or umbrellas) in outdoors. Older age, lower socioeconomic status, including income and education, as well as UVB exposure were main factors of high prevalence of pterygium in our study.

Obesity is an important risk factor for diabetes. $\mathrm{HbA} 1 \mathrm{C}$ is a marker of the average blood glucose level over the $2 \sim 3$ months prior to measurement. In some previous studies, the researchers did not detect associations between the pterygium prevalence and DM[9, 17, 24, 31]. In those studies, DM were recorded by self-reported lack of detailed examination and blood measurement. In recent studies, some parameters like height, weight, WC, lipid, glucose and HbA1c were measured[12].Nam et al showed obese was associated the pterygium prevalence in women and high BMI and WC were risk factors[22]. Serum Immunoglobulin E and serum 25-hydroxyvitamin were associated with the pterygium in Korean adults [32, 33]. In our study, we found higher $\mathrm{BMI}$ and $\mathrm{HbA} 1 \mathrm{c}$ were risk factors for women, not for men. Diabetes and associated factors maybe contributed to higher prevalence in women with D2M in our study. Further studies are needed to examine the gender difference in the pathogenesis of pterygium for obese and diabetes subjects.

The correlation of pterygium prevalence with gender is controversial. Some studies found that gender was not associated with pterygium[12, 18, 19,31]. Some studies found women was a protective factor of pterygium[6, 8, 26, 34, 35]. A recently study showed reproductive factors that increase estrogen exposure have protective effects against pterygium in females[36]. Other studies showed women was a risk factor of the pterygium prevalence $[13,17,30]$.A meta-analysis showed the presence of pterygium in China was also more frequently in men than in women[15]. Yang's meta-analysis showed the presence of pterygium is higher in man in east area and in women in west area in China[23]. Our result agreed with 2 studies from rural Dali and Tibetan West China[5, 17]. The prevalence of pterygium was higher in women $(24.43 \%)$ than in men $(18.32 \%)$ with D2M in Funing. Differences in lifestyles and the culture-related gender-specific daily activities between the various populations may be the reasons for the variations in the association between the pterygium prevalence and gender. Nam et al showed obese was 
associated the pterygium prevalence in women and high BMI and WC are risk factors[22]. In our study, we found higher BMI and $\mathrm{HbA} 1 \mathrm{c}$ were risk factors for women, not for men. Diabetes and associated factors maybe contributed to higher prevalence in women with D2M. Further studies are needed to examine the gender difference in the pathogenesis of pterygium for obese and diabetes subjects.

Cigarette smoking is an important public health problem. Smoking was associated with eye diseases such as hyperopia and age-related nuclear cataract[37]. The association between smoking and pterygium remained unclear and contradictory. Some studies reported that smoking was an independent protective factor against pterygium after adjusting multiple risk factors for many studies $[4,9,20,26,38,39]$. Cigarette smoking (current or ever smoked) was associated with a reduced risk of pterygium, especially in current smokers[26, 40]. Some studies showed the opposite results that cigarette smoking was a risk factor[41, 42]. Some studies did not find the association between smoking and pterygium[17, 19]. Nevertheless, a meta-analysis study showed that smoking was a protective factor for pterygium, especially in current smokers[40]. Our results supported smoking also was an independent protective factor of pterygium in D2M patients, especially in male patients currently smoking. Up to now, the exact molecular mechanism for the protective effect of smoking on pterygium is not known. The protective effect might be related with suppression the expression of inflammatory mediator and alteration of the component of tear film, which was still lack of relevant experimental data[43, 44]. Investigations are needed to unveil its molecular basis serving therapeutic purposes.

The association between hypertension and pterygium was not uncertain. Some studies found no association between hypertension and pterygium[19]. Studies in Yunnan in China and South Korea showed hypertension was a risk factor of pterygium[10,17]. Studies of the Singapore Malay Eye Study and Hebei in China reported on an increased pterygium prevalence with higher systolic blood pressure[8, 20]. In our study, our results agreed that hypertension was an independent risk factor of pterygium in our diabetic population.

Sunlight is the common casual factors of several eye diseases including age-related cataract and pterygium, which also be called sunlight-related eye diseases[45]. However, the association between cataract and pterygium was still unclear. Our study found opacity of lens nucleus grade over 2 was an independent risk factors of pterygium in D2M population, which was agree with the result in healthy population in the Ural Eye and Medical study[12]. However, no association was found between pterygium and the incidence of cortical, nuclear, or posterior subcapsular cataract in the Blue Mountains Eye Study[46]. Smoking is a strong risk factor for age-related nuclear cataract[37]. This result needs to be further verified by other studies.

\section{Limitations of the study}

Nevertheless, these limitations must be considered when interpreting the results of this study. (1) The participants enrolled in our study were only D2M patients 50 years and above registered in Funing Country Center for Chronic Disease Control and Prevention, type 1 diabetes patients were not included. Some subjects with prediabetes and newly diagnosed diabetes may be missed in our study. (2) The pterygium was diagnosed by slit lamp microscope, but not graded. (3) The time of sunlight exposure did not record exact hours, instead of whether work in outdoors and exposure sunlight over 5 hours or less. (4) The participants were only detected fast blood glucose and HbA1c, but not other blood biochemical indexes.

\section{Conclusion}

In conclusion, the pterygium prevalence was rather higher in the aged rural population with D2M in Jiangsu, China. Age and women were positively, and current smoking negatively associated with the prevalence of pterygium. However, higher BMI and HA1C maybe increase the prevalence of pterygium in female D2M patients. Proper protection against sun exposure using spectacles, brimmed hats, and appropriate headgear, especially for outdoor famers, would help to reduce disease risk. Public education focusing on the avoidance of unnecessary sunlight exposure could reduce the development of pterygium.

\section{Declarations}

Page $10 / 15$ 


\section{Acknowledgments}

We thank the participants of this study. We also thank faculties of Funing Country Center for Chronic Disease Control and Prevention For continuous support, assistance, and cooperation.

\section{Contributors}

ZJF and QB contributed equally to the paper. ZJF, QB and YM performed the statistical analysis and drafted the manuscript. ZRR conceived, designed, and supervised the study. ZJF, QB, WSY, LBH, SDJ, LCK AND SYQ conducted the epidemiological survey. All authors contributed to acquisition, analysis, or interpretation of data. All authors revised the report and approved the final version before submission.

\section{Funding}

The study was supported by grants from the Science and Technology Project of Nantong Municipality, Jiangsu Province, China (JC2019078, MS12019030).

\section{Ethics approval}

The study was conducted in accordance with the tenets of the Declaration of Helsinki and approved by the Ethics Committee of Affiliated Hospital of Nantong University (No.2014-071). All participants signed the informed consent forms after fully informed of the purpose and procedures.

\section{DECLARATION OF INTEREST}

The authors report no conflicts of interest. The authors alone are responsible for the content and writing of the paper.

\section{ORCID iD}

Ronggong Zhu https://orcid.org/0000-0002-3519-7566

Junfang Zhang https://orcid.org/0000-0002-4056-6928

\section{References}

1. Li Y, Teng D, Shi X, Qin G, Qin Y, Quan H, Shi B, Sun H, Ba J, Chen B et al (2020) Prevalence of diabetes recorded in mainland China using 2018 diagnostic criteria from the American Diabetes Association: national cross sectional study. BMJ 369:m997

2. Cui Y, Zhang L, Zhang M, Yang X, Zhang L, Kuang J, Zhang G, Liu Q, Guo H, Meng Q (2017) Prevalence and causes of low vision and blindness in a Chinese population with type 2 diabetes: the Dongguan Eye Study. Sci Rep 7(1):11195

3. McCarty CA, Fu CL, Taylor HR (2000) Epidemiology of pterygium in Victoria, Australia. Br J Ophthalmol 84(3):289-292

4. Gazzard G, Saw SM, Farook M, Koh D, Widjaja D, Chia SE, Hong CY, Tan DT (2002) Pterygium in Indonesia: prevalence, severity and risk factors. Br J Ophthalmol 86(12):1341-1346

5. Lu P, Chen X, Kang Y, Ke L, Wei X, Zhang W (2007) Pterygium in Tibetans: a population-based study in China. Clin Exp Ophthalmol 35(9):828-833

6. Ma K, Xu L, Jie Y, Jonas JB (2007) Prevalence of and factors associated with pterygium in adult Chinese: the Beijing Eye Study. Cornea 26(10):1184-1186

7. Fotouhi A, Hashemi H, Khabazkhoob M, Mohammad K (2009) Prevalence and risk factors of pterygium and pinguecula: the Tehran Eye Study. Eye 23(5):1125-1129

8. Cajucom-Uy H, Tong L, Wong TY, Tay WT, Saw SM (2010) The prevalence of and risk factors for pterygium in an urban Malay population: the Singapore Malay Eye Study (SiMES). Br J Ophthalmol 94(8):977-981 
9. Marmamula S, Khanna RC, Rao GN (2013) Population-based assessment of prevalence and risk factors for pterygium in the South Indian state of Andhra Pradesh: the Andhra Pradesh Eye Disease Study. Investig Ophthalmol Vis Sci 54(8):5359-5366

10. Pyo EY, Mun GH, Yoon KC (2016) The prevalence and risk factors for pterygium in South Korea: the Korea National Health and Nutrition Examination Survey (KNHANES) 2009-2010. Epidemiol health 38:e2016015

11. Malekifar P, Esfandiari H, Behnaz N, Javadi F, Azish S, Javadi MA, Kalantarion M (2017) Risk Factors for Pterygium in Ilam Province, Iran. J ophthalmic Vis Res 12(3):270-274

12. Bikbov MM, Zainullin RM, Kazakbaeva GM, Gilmanshin TR, Salavatova VF, Arslangareeva II, Nikitin NA, Panda-Jonas S, Zaynetdinov AF, Kazakbaev RA et al (2019) Pterygium Prevalence and Its Associations in a Russian Population: The Ural Eye and Medical Study. Am J Ophthalmol 205:27-34

13. Lin YH, Sun CC, Yeung L, Yu YW, Sun MH, Chen KJ (2019) Epidemiologic study of pterygium in Taiwan. Jpn J Ophthalmol 63(4):297-303

14. Fernandes AG, Salomao SR, Ferraz NN, Mitsuhiro MH, Furtado JM, Munoz S, Cypel MC, Cunha CC, Vasconcelos GC, Sacai PY et al (2020) Pterygium in adults from the Brazilian Amazon Region: prevalence, visual status and refractive errors. $\mathrm{Br} J$ Ophthalmol 104(6):757-763

15. Rezvan F, Khabazkhoob M, Hooshmand E, Yekta A, Saatchi M, Hashemi H (2018) : Prevalence and risk factors of pterygium: a systematic review and meta-analysis. Survey of ophthalmology 63(5):719-735

16. Alqahtani JM (2013) The prevalence of pterygium in Alkhobar: A hospital-based study. J Fam Commun Med 20(3):159161

17. Zhong H, Cha X, Wei T, Lin X, Li X, Li J, Cai N, Li J, Su X, Yang Y et al (2012) Prevalence of and risk factors for pterygium in rural adult chinese populations of the Bai nationality in Dali: the Yunnan Minority Eye Study. Investig Ophthalmol Vis Sci 53(10):6617-6621

18. Chen T, Ding L, Shan G, Ke L, Ma J, Zhong Y (2015) Prevalence and racial differences in pterygium: a cross-sectional study in Han and Uygur adults in Xinjiang, China. Investig Ophthalmol Vis Sci 56(2):1109-1117

19. Jiao W, Zhou C, Wang T, Yang S, Bi H, Liu L, Li Y, Wang L (2014) Prevalence and risk factors for pterygium in rural older adults in Shandong Province of China: a cross-sectional study. Biomed Res Int 2014:658648

20. Pan Z, Cui J, Shan G, Chou Y, Pan L, Sun Z, Cui Z, Sun J, Cao Y, Zhao J et al (2019) Prevalence and risk factors for pterygium: a cross-sectional study in Han and Manchu ethnic populations in Hebei, China. BMJ open 9(2):e025725

21. Zhao L, You QS, Xu L, Ma K, Wang YX, Yang H, Jonas JB (2013) 10-year incidence and associations of pterygium in adult Chinese: the Beijing Eye Study. Investig Ophthalmol Vis Sci 54(2):1509-1514

22. Nam GE, Kim S, Paik JS, Kim HS, Na KS (2016) Association between pterygium and obesity status in a South Korean population. Medicine 95(50):e5664

23. Yang M, Kang LH, Guan Y, Qin B, Zhang JF (2019) Meta-analysis of prevalence of pterygium among people aged over 40 in China. [Zhonghua Shiyan Yanke Zazhi] Chinese Journal of Experimental Ophthalmology 37(3):190-196

24. Zhang JF, Yang M, Zhu RR, Qin B, Shi HH, Kang LH, Shi J, Hu N, Guan HJ (2019) Prevalence and associated factors for pterygium in rural people aged 50 years and above in Fuming Country, Jiangsu Province. [Zhonghua Shiyan Yanke Zazhi] 37(3):212-217

25. Zhao J, Ellwein LB, Cui H, Ge J, Guan H, Lv J, Ma X, Yin J, Yin ZQ, Yuan Y et al (2010) Prevalence of vision impairment in older adults in rural China: the China Nine-Province Survey. Ophthalmology 117(3):409-416 416 e401

26. Sun LP, Lv W, Liang YB, Friedman DS, Yang XH, Guo LX, Peng Y, Wang NL, Wang JJ (2013) The prevalence of and risk factors associated with pterygium in a rural adult Chinese population: the Handan Eye Study. Ophthalmic Epidemiol 20(3):148-154

27. Wang Y, Shan G, Gan L, Qian Y, Chen T, Wang H, Pan X, Wang W, Pan L, Zhang X et al (2020) Prevalence and associated factors for pterygium in Han and Mongolian adults: a cross-sectional study in inner Mongolian, China. BMC Ophthalmol 20(1):45

Page 12/15 
28. Pan ZX, Shan GL, Wang XJ, Pan L, Tao ZY, He HJ, Ma J, Ren XL, Zhao HJ, Zhong Y (2020) : [Prevalence and risk factors of pterygium in Han and Yugur populations in Gansu Province, China]. [Zhonghua yan ke za zhi] Chinese journal of ophthalmology 56(8):600-607

29. Detels R, Dhir SP (1967) Pterygium: a geographical study. Arch Ophthalmol 78(4):485-491

30. Rim TH, Kang MJ, Choi M, Seo KY, Kim SS (2017) The incidence and prevalence of pterygium in South Korea: A 10-year population-based Korean cohort study. PLoS ONE 12(3):e0171954

31. Asokan R, Venkatasubbu RS, Velumuri L, Lingam V, George R (2012) Prevalence and associated factors for pterygium and pinguecula in a South Indian population. Ophthalmic \& physiological optics: the journal of the British College of Ophthalmic Opticians 32(1):39-44

32. Chun YH, Paik JS, Oh JH, Kim HS, Na KS (2018) Association between pterygium, sun exposure, and serum 25hydroxyvitamin in a nationally representative sample of Korean adults. Lipids Health Dis 17(1):260

33. Yoo TK, Kim SW, Seo KY (2018) Association between Serum Immnunoglobulin E and Pterygium: A Population-Based Study from South Korea. Curr Eye Res 43(9):1090-1096

34. Ang M, Li X, Wong W, Zheng Y, Chua D, Rahman A, Saw SM, Tan DT, Wong TY (2012) Prevalence of and racial differences in pterygium: a multiethnic population study in Asians. Ophthalmology 119(8):1509-1515

35. Shiroma H, Higa A, Sawaguchi S, Iwase A, Tomidokoro A, Amano S, Araie M (2009) Prevalence and risk factors of pterygium in a southwestern island of Japan: the Kumejima Study. Am J Ophthalmol 148(5):766-771e761

36. Shin E, Lim DH, Chung TY, Han G, Yoo JE, Jeon KH, Han K, Shin DW (2021) Reproductive risk factors of pterygium in postmenopausal women: a nationwide study in the Republic of Korea. Sci Rep 11(1):12473

37. Nita M, Grzybowski A (2017) Smoking and Eye Pathologies. A Systemic Review. Part I. Anterior Eye Segment Pathologies. Curr Pharm Design 23(4):629-638

38. West S, Munoz B (2009) Prevalence of pterygium in Latinos: Proyecto VER. Br J Ophthalmol 93(10):1287-1290

39. Rim TH, Nam J, Kim EK, Kim TI (2013) Risk factors associated with pterygium and its subtypes in Korea: the Korean National Health and Nutrition Examination Survey 2008-2010. Cornea 32(7):962-970

40. Rong SS, Peng Y, Liang YB, Cao D, Jhanji V (2014) : Does cigarette smoking alter the risk of pterygium? A systematic review and meta-analysis. Investigative ophthalmology \& visual science 55(10):6235-6243

41. Li Z, Cui H (2013) Prevalence and associated factors for pterygium in a rural adult population (the Southern Harbin Eye Study). Cornea 32(6):806-809

42. Wong TY, Foster PJ, Johnson GJ, Seah SK, Tan DT (2001) The prevalence and risk factors for pterygium in an adult Chinese population in Singapore: the Tanjong Pagar survey. Am J Ophthalmol 131(2):176-183

43. Goncalves RB, Coletta RD, Silverio KG, Benevides L, Casati MZ, da Silva JS, Nociti FH Jr (2011) Impact of smoking on inflammation: overview of molecular mechanisms. Inflamm research: official J Eur Histamine Res Soc [et al] 60(5):409424

44. Rom O, Avezov K, Aizenbud D, Reznick AZ (2013) Cigarette smoking and inflammation revisited. Respir Physiol Neurobiol 187(1):5-10

45. Young RW (1994) The family of sunlight-related eye diseases. Optometry and vision science: official publication of the American Academy of Optometry 71(2):125-144

46. Pham TQ, Wang JJ, Rochtchina E, Mitchell P (2005) Pterygium, pinguecula, and 5-year incidence of cataract. Am J Ophthalmol 139(6):1126-1128

\section{Figures}




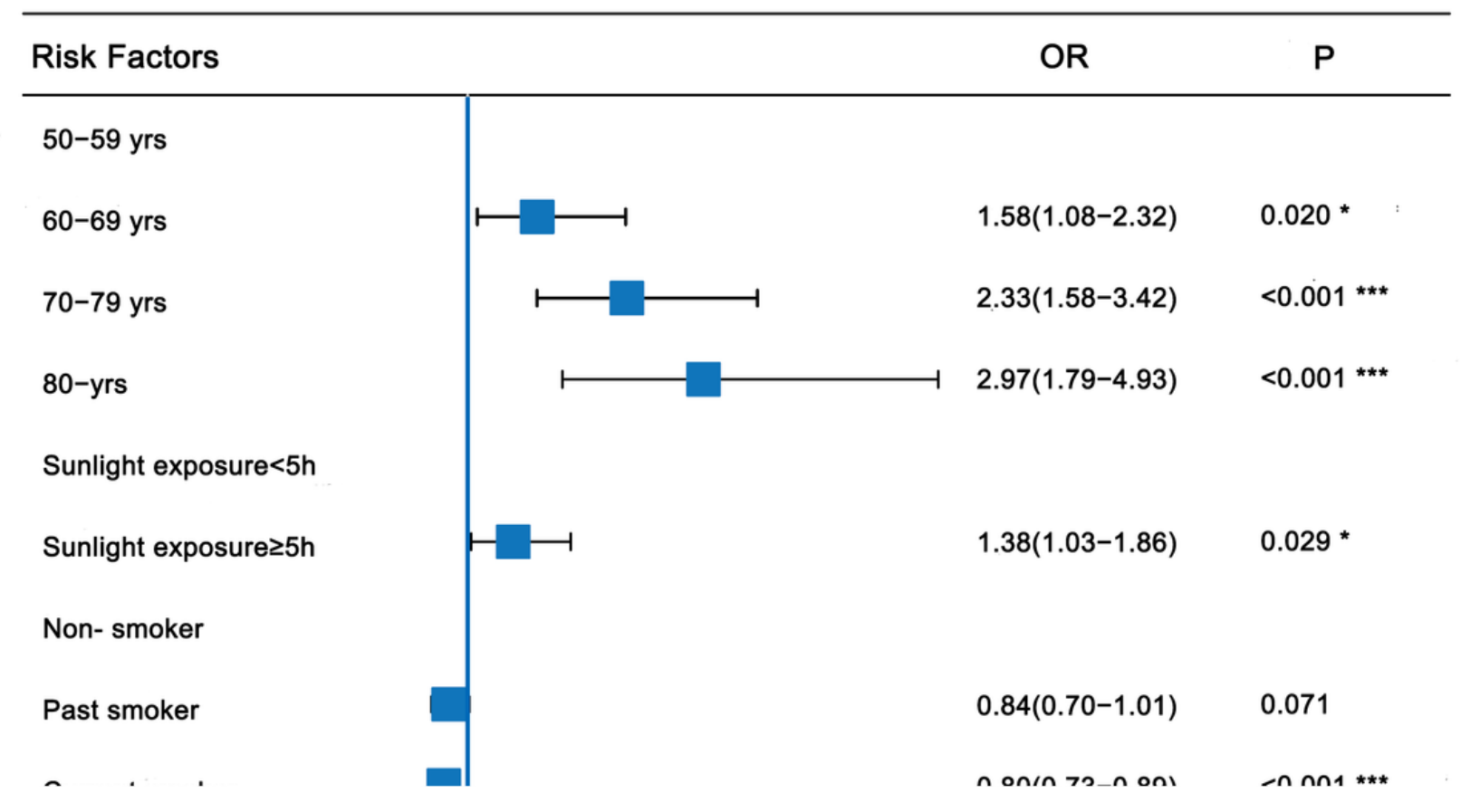

Figure 1

Multivariate logistic regression analysis for factors associated with pterygium. 
Male

Female

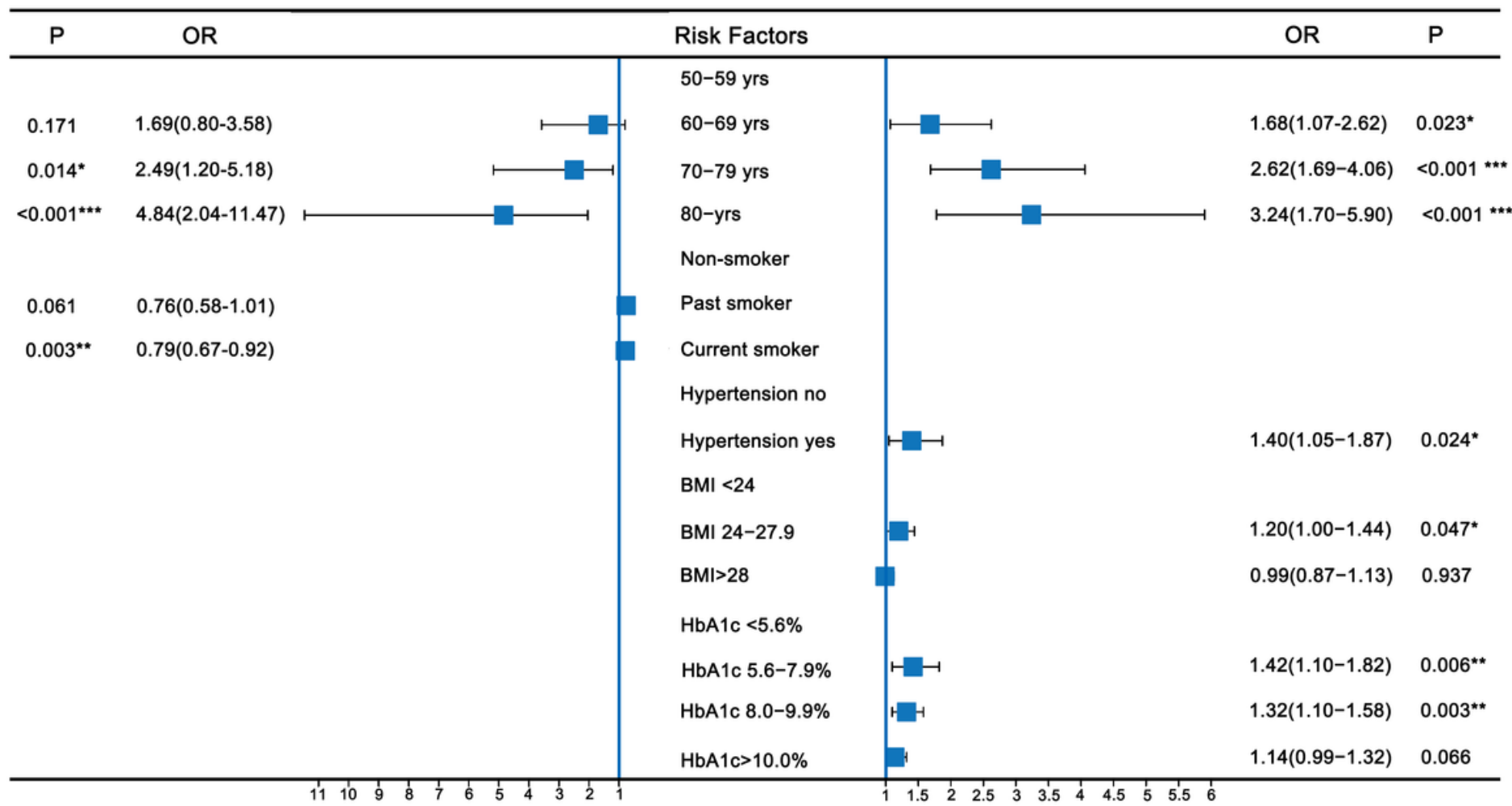

\section{Figure 2}

Multivariate logistic regression analysis for factors associated with pterygium in subgroup of male and female participants. 